Nouveaux visages de l'anglais de spécialité : objectifs, domaines, approches et outils de demain

\title{
L'anglais de spécialité en chimie organique : entre indétermination terminologique et multidimensionnalité
}

\section{Sandrine Peraldi}

\section{CpenEdition}

\section{Journals}

Édition électronique

URL : http://journals.openedition.org/asp/3066

DOI : 10.4000/asp.3066

ISSN : 2108-6354

\section{Éditeur}

Groupe d'étude et de recherche en anglais de spécialité

Édition imprimée

Date de publication : 15 novembre 2012

Pagination : 5-24

ISSN : 1246-8185

\section{Référence électronique}

Sandrine Peraldi, «L'anglais de spécialité en chimie organique : entre indétermination terminologique et multidimensionnalité ", ASp [En ligne], 62 | 2012, mis en ligne le 07 octobre 2014, consulté le 02 novembre 2020. URL : http://journals.openedition.org/asp/3066 ; DOI : https://doi.org/10.4000/asp. 3066

Ce document a été généré automatiquement le 2 novembre 2020.

Tous droits réservés 


\title{
L'anglais de spécialité en chimie organique : entre indétermination terminologique et multidimensionnalité
}

\author{
Sandrine Peraldi
}

\section{Problématique et cadre théorique}

1 Les observations exposées dans la présente étude relèvent de recherches menées en terminologie et en linguistique de corpus et visent à apporter un certain nombre d'éléments nouveaux sur une langue de spécialité qui a été relativement peu étudiée jusqu'à présent, du moins sur un plan discursif et non sur un plan nomenclatural, à savoir la langue de la chimie. Par ailleurs, la deuxième spécificité de ces travaux réside dans la mise en évidence de deux autres phénomènes linguistiques qui ont également constitué un parent pauvre en terminologie : l'indétermination terminologique et la multidimensionnalité. C'est en effet à travers l'analyse de ces deux thématiques spécifiques qu'un certain nombre de tendances ou de régularités au sein de l'anglais de la chimie ont pu être dégagées. Aussi, nous avons choisi, dans le présent article, de reproduire le cheminement intellectuel qui nous a menée vers ces découvertes d'ordre linguistique et terminologique : nous commençons par évoquer les postulats théoriques et épistémologiques qui ont été les nôtres, pour aborder ensuite la méthodologie adoptée et enfin exposer le cœur de notre analyse et ses incidences sur la langue de spécialité en chimie. 


\subsection{Dichotomie épistémologique et linguistique entre sciences dures et sciences douces}

Pour resituer rapidement le cadre théorique ainsi que les objectifs de ces travaux de recherche, il s'agissait de rééquilibrer, pour ne pas dire remettre en question, une certaine propension à la généralisation en terminologie, et plus spécifiquement la dichotomie existant communément entre sciences dures et sciences douces, d'une part, et entre domaines techniques et scientifiques, d'autre part. En effet, étant donné que toute recherche scientifique vise a priori à établir un ensemble de certitudes en matière de connaissances et de catégorisation, on s'attend à ce que les langages appelés à désigner cette recherche soient tout aussi rigoureux et exacts, mésestimant souvent les difficultés d'ordre conceptuel et linguistique susceptibles d'apparaître dans ces domaines, notamment à l'aune des sciences humaines et sociales.

Sur un plan épistémologique, les sciences douces seraient en effet moins authentiquement scientifiques, plus imprécises, dominées par l'idéologie et, de ce fait, sujettes aux évolutions culturelles et interculturelles. Inversement, les sciences exactes, dans la mesure où elles explorent des systèmes axiomatiques et décrivent des concepts tangibles et objectaux, ne laissent a priori aucune place à l'approximation scientifique et langagière :

Dans les autres sciences, on admet qu'il existe une vérité et une seule. L'activité scientifique consiste à rechercher les lacunes des vérités établies afin de les remplacer par des théories nouvelles qui comblent ces lacunes. [...] Rien de tel en économie où des thèses contradictoires continuent à être enseignées et approfondies [...]. L'économie se présente comme un hybride improbable de rigueur scientifique (prétendument garantie par l'usage des mathématiques) et de philosophie [...]. La raison essentielle me semble être que l'économie, science humaine et sociale, est encore dominée par l'idéologie. (Dréan 2001) cité par Rogel (2007)

Sur un plan linguistique cette fois, la terminologie, ou du moins la théorisation de la terminologie, s'est d'abord construite, au travers des travaux d'Eugen Wüster (1968), à partir d'une expérience dans les domaines techniques (celle de la machine-outil) et donc autour d'une activité spéculative pour les sciences. E. Wüster supposait, en effet, que les deux démarches - technologique et scientifique - étaient les mêmes pour la terminologie, citant d'ailleurs à plusieurs reprises Lavoisier, l'un des pères fondateurs de la première nomenclature en chimie. Aussi, un certain nombre de terminologues (Gambier 1991; Weissenhofer 1995; Otman 1996) estiment que les domaines scientifiques, au même titre que les disciplines techniques, se prêtent plus aisément à un traitement terminologique et notamment à une approche conceptuelle et logique des unités linguistiques, contrairement aux disciplines présentant une forte dimension évaluative, à savoir des domaines tributaires de l'interprétation de chaque individu et se traduisant par conséquent par des concepts fluctuants :

À ceci près que, mis à part des domaines comme la botanique, la zoologie ou la chimie, hautement structurés de manière taxinomique, les domaines inter- et transdisciplinaires, les domaines en constitution et en évolution, les disciplines à la recherche d'une légitimation et d'une valorisation, c'est-à-dire toutes celles qui intéressent les terminologues, ne proposent qu'un découpage flou de leur "substance » en classes et sous-classes. Cette indétermination se reflète dans les formes linguistiques de classification employées qui ne sont en fait, que des indices faibles de classification (genre de, espèce de, sorte de, type de...). (Otman 1996 : 48) 


\subsection{Indices d'indétermination}

Ce postulat épistémologique et terminologique est d'autant plus attesté en chimie qu'il s'agit d'un domaine technico-scientifique pragmatique, doté d'une nomenclature standard et mondialement reconnue, permettant de réglementer et de systématiser les désignations officielles des substances chimiques. La chimie est effectivement l'une des rares disciplines à bénéficier de nombreuses et constantes activités normalisatrices, parmi lesquelles la publication de recommandations systématiques et de glossaires terminologiques destinés à codifier les pratiques langagières acceptables. Or divers indices préliminaires d'ordre linguistique et documentaire indiquent au contraire que la communication entre experts, mais aussi entre spécialistes et non-initiés, est souvent floue, ambiguë, ce qui porte préjudice à la structuration conceptuelle et à la transmission des connaissances spécialisées au sein de la discipline. Nous proposons de passer en revue quelques-uns de ces indicateurs.

\section{L'absence de ressources terminologiques fiables}

6 La majorité des ouvrages se caractérisent en effet par des mises à jour extrêmement disparates, des données terminologiques éparpillées et incomplètes, une absence totale de structuration conceptuelle et un recouvrement partiel des concepts-clés du domaine, laissant des vides importants dans la représentation des connaissances, à l'image du traitement proposé par le IUPAC Gold Book considéré comme le référentiel terminologique en chimie :

Nevertheless, a user will find frustrating discrepancies in coverage. For example, buffer capacity is defined, but the more fundamental term buffer is not; spectroscope, spectrograph, and spectrometer are defined, but spectrophotometer is not; bond and covalent bond are defined, but ionic bond is not. (Dehne $1997: 349$ )

Unfortunately, it is clear from the Compendium that there is no standard practice in IUPAC for the writing of standard definitions; many are well designed, many others are not. A large number of definitions use more than one sentence. Often it is not clear at all where the defining sentence(s) leaves off and descriptive material begins. Some symbols are included in the body of the text, some are not. Some entries are small encyclopedia articles. Some definitions begin with a verb; some are constructed in the form of "if... then... (term)"; some include the term in the definition. Of course, there are definitions that are combinations of these flawed structures. (Dehne 1997 : 350)

La présence significative de concepts abstraits, et plus spécifiquement de notions technologiques

7 Selon la définition proposée par Allal Assal (1994: 460-461), ces dernières constituent des notions chargées de théorie. Elles ne renvoient pas à des objets matériels et observables, mais à des principes et des méthodes théoriques dont on peut uniquement observer le déroulement et le résultat, à l'image notamment des mécanismes réactionnels de la chimie.

\section{Le caractère empratique du discours de spécialité de la chimie (Lefèvre 2004)}

Selon le linguiste, le propre du discours de nature scientifique et technique est d'être elliptique : les dialogues sont réduits au strict minimum entre experts tandis que les descriptions reposent sur des schémas et des symboles dans lesquels figurent les termes strictement nécessaires à la démonstration. En réalité, Michel Lefèvre s'appuie ici sur les propos de Karl Bühler (1934) selon qui l'ellipse peut s'expliquer dans la langue par l'absence de nécessité, dans une situation de communication donnée, 
d'inclure l'ensemble des éléments récursifs. Autrement dit, le déroulement d'une activité quelconque peut s'effectuer sans intervention du langage tant que son déroulement est connu, prévisible. Mais dès lors que la continuité de l'activité impose de préciser un choix, de réorienter son cours, en d'autres termes lorsque les participants de l'activité atteignent un point de diacrise, le discours empratique intervient, notamment sous la forme d'îlots de parole :

Des îlots de parole apparaissent au milieu de la mer des échanges silencieux mais univoques aux endroits où il faut une différenciation, une diacrise, un choix entre plusieurs possibilités, ce pour quoi il suffit d'un mot. Ces îlots apparaissent et sont les bienvenus comme des noms et des flèches sur les panneaux indicateurs sont bienvenus à la croisée des chemins que l'on emprunte. (Bühler 1934 : 39)

9 Par conséquent, seuls le cotexte et le contexte situationnel (extralinguistique) permettent de comprendre l'ellipse. Or, la communication entre chimistes organiciens se fait précisément par les deux biais évoqués précédemment: d'une part, à travers l'utilisation concomitante de structures et d'équations chimiques afin d'illustrer le point de vue de l'émetteur et, d'autre part, par la capacité de l'interlocuteur de comprendre les propos de l'autre grâce au contexte, c'est-à-dire dans un cadre de communication d'expert à expert. Par conséquent, la langue de la chimie est particulièrement opaque, tout en laissant trop de place à l'interprétation (Krief et al. 2008: 205), portant préjudice à la transmission des connaissances, qu'il s'agisse de néophytes ou d'initiés en la matière.

Enfin, l'on observe l'existence d'un certain nombre de variantes terminologiques au sein même de la nomenclature chimique associée à plusieurs niveaux de communication (formel, semi-formel et informel) en fonction des locuteurs et des situations d'énonciation, ce qui se traduit par une prolifération de noms triviaux et semi-systématiques qui concurrencent directement les désignations officielles (Loening 1986 : 93 ; Sager 1990 : 97 ; Godly 1993 : 161).

11 Bref, un ensemble d'éléments qui témoigne du fait que les nomenclatures en particulier, et les vocabulaires normés en général, ne sont pas uniquement désignationnels, mais qu'ils assument également une fonction interlocutive et dialogique. Ils doivent donc, selon les propres termes de François Gaudin (2003: 72), être inclus dans le fonctionnement social et éventuellement conflictuel des langues.

En l'occurrence, cette problématique de communication défaillante émane directement d'un laboratoire de chimie organique (des Facultés universitaires Notre-Dame de la Paix à Namur). Elle répond à un besoin spécifique de communication énoncé par des chimistes organiciens, ce qui a permis d'ancrer ces travaux dans une véritable démarche de linguistique appliquée, à travers la construction d'une ressource ontoterminologique spécifique en chimie (Krief \& Castillo-Colaux 2005). Il a donc été décidé d'éprouver, à grande échelle, le caractère indéterminé de la chimie organique, tout en cherchant à accroître un certain nombre de connaissances s'agissant des spécificités lexicales, morphosyntaxiques, néologiques et stylistiques de ce discours spécialisé. 


\section{Méthodologie}

\subsection{Exploration textuelle}

13 Sur un plan méthodologique, nous avons procédé à l'exploration textuelle outillée (via le concordancier AntConc) d'un double corpus - pédagogique et spécialisé - de près d'1,5 million de mots, plaçant de fait notre démarche de recherche dans le cadre théorique et applicatif de la linguistique de corpus. Nous estimons en effet que toute analyse linguistique doit systématiquement reposer sur l'observation de données linguistiques authentiques dans leur environnement naturel. Le corpus fournit non seulement des attestations des unités lexicales, mais il permet également d'observer la langue et les termes « in vivo " (Enguehard $2005: 274$ ), ce qui induit une connaissance approfondie des pratiques langagières en langue de spécialité, associée à une compréhension plus fine du domaine à l'étude. Restreindre les langues de spécialité à une analyse notionnelle serait en effet largement réducteur.

Il ne semble plus possible de s'intéresser à la langue dans ce qu'elle a de systématique sans rendre compte en même temps des situations réelles dans lesquelles les règles ainsi élaborées se réalisent, c'est-à-dire sans déterminer la sphère d'application de ces règles et leurs conditions de variation. La linguistique de corpus apparaît bien alors comme le meilleur moyen d'atteindre la diversité des faits de langue authentiques. (Jacques $2005: 25$ )

À l'instar des travaux de Danielle Candel (2001: 18), il a été décidé de donner une double direction à la collecte du corpus, à la fois par souci de représentativité et d'exhaustivité des données terminologiques. En effet, la nature des informations glanées dans un texte est fortement tributaire à la fois du cadre de communication (Pearson 1998: 36) et du genre textuel (Swales 1990: 58) dont il relève. Aussi, un premier corpus a été compilé sur la base de textes dits avancés, caractérisés par un niveau élevé de technicité et de spécialisation. Ils correspondent donc à un cadre de communication d'expert à expert, lequel se caractérise entre autres par une très forte densité terminologique, technique et phraséologique, mais un nombre négligeable de données d'ordre définitoire. Le genre textuel sélectionné est celui des articles de recherche. Par ailleurs, un second ensemble textuel a été assemblé, comprenant cette fois des textes à caractère instructif, c'est-à-dire pédagogiques ou éducatifs et correspondant à un cadre de communication d'expert à initié. Ce corpus pédagogique a permis de servir trois desseins spécifiques : aider à appréhender, en début de projet, le domaine extrêmement complexe de la chimie organique ; fournir le matériel définitoire et conceptuel en vue d'une analyse comparative; enfin, compléter les éléments définitoires et notionnels qui serviront à la compilation du dictionnaire que nous projetons de réaliser.

Tableau 1. Corpus

\begin{tabular}{|l|l|l|}
\hline & $\begin{array}{l}\text { Corpus } \\
(\mathrm{CS})\end{array}$ & spécialisé \\
\hline
\end{tabular}




\begin{tabular}{|l|l|l|}
\hline $\begin{array}{l}\text { Corpus } \\
\text { original }\end{array}$ & $\begin{array}{l}\text { 210 articles (PDF) } \\
1693477 \text { mots }\end{array}$ & $\begin{array}{l}\text { 1) The Organic Chemistry Textbook } \\
\text { 2) Virtual Textbook of Organic Chemistry } \\
\text { 3) Organic Chemistry On-Line Learning Center (Chiffre non } \\
\text { pertinent) }\end{array}$ \\
\hline $\begin{array}{l}\text { Corpus trié } \\
\text { (non nettoyé) }\end{array}$ & 1327639 mots & articles (PDF) \\
\hline Cófinitif & 832321 mots & $\begin{array}{l}\text { 1) } 214967 \text { mots } \\
\text { 2) } 310910 \text { mots } \\
\text { 3) } 161213 \text { mots } \\
\text { 686 } 790 \text { mots }\end{array}$ \\
\hline
\end{tabular}

15 Eu égard à la masse de données à analyser, nous avons eu recours à des critères sémantiques de conservation et de filtrage terminologique (Condamines \& Rebeyrolle $2000: 7$ ) et des marqueurs linguistiques spécifiques (Pearson 1998: 66-104; Candel 2000 : 55 ; L'Homme $2004: 156$; Aussenac-Gilles \& Condamines 2009).

\subsection{Analyse componentielle}

Sur un plan analytique, il est apparu clairement, notamment à l'aune des travaux de Philippe Thoiron, Pierre Arnaud, Henri Béjoint et Claude-Pierre Boisson (1996: 512-524), de Rita Temmerman (2000) et de Judit Freixa, Sabela Silva et Maria Teresa Cabré (2008 : 731-744), qu'une analyse componentielle revisitée et corrigée des énoncés définitoires de certains termes clés constituait un axe majeur de recherche et surtout de validation des phénomènes d'indétermination et de multidimensionnalité. Bien que la seule représentation du concept comme ensemble de traits sémantiques soit insuffisante ${ }^{1}$ - c'est d'autant plus vrai qu'il a été largement prouvé que certaines notions de structure prototypique n'autorisaient pas de définition classique par traits nécessaires et suffisants - l'analyse sémique nous est néanmoins apparue, sur un plan heuristique, comme étant un outil descriptif majeur :

There can be no semantic description without some kind of decompositional analysis: it is hard to conceive any form of comparative analysis that does not involve breaking down the comparanda into components and characteristics. (Geeraerts et al. 1994 : 38)

17 Par conséquent, nous avons procédé à une décomposition des traits sémantiques et des relations conceptuelles caractérisant certains concepts clés de la chimie par le biais d'une analyse définitoire comparative. Autrement dit, l'information sémantique exprimée dans les différentes définitions des termes à l'étude a été décomposée, examinée et comparée afin de déterminer quels traits sémantiques transparaissent dans chaque énoncé définitoire et de quelle manière les termes se positionnent dans le réseau conceptuel du domaine. 


\section{Analyse définitoire comparative et indétermination}

\subsection{Indétermination terminologique}

18 L'analyse définitoire et componentielle décrite précédemment s'est également accompagnée d'une comparaison des perspectives d'approche de la chimie et de leurs incidences sur la formulation des définitions ainsi que d'une étude sur les liens conceptuels et les relations associatives, dans une tentative de représentation des connaissances du domaine. À notre sens, ces trois axes analytiques ont permis d'identifier clairement le caractère indéterminé de certaines unités de la chimie, tout en démontrant les faiblesses de l'approche classique pour rendre compte de ce type de phénomènes. Cette recherche a non seulement permis de mettre en évidence un manque de consensus global s'agissant de l'hyperonyme et des traits conceptuels saillants des concepts à l'étude, mais également un niveau descriptif extrêmement vague et imprécis. Par indétermination terminologique, nous entendons le caractère flou ou imprécis des concepts ou des définitions qui les représentent (Andersen 2002, 2007). Plus précisément, un concept est considéré comme indéterminé, soit lorsque son extension est vague, soit lorsqu'il est impossible de déterminer si l'objet décrit appartient à la classe désignée par le concept. En d'autres termes, lorsque le signifié est véritablement imprécis, non clairement défini, quel que soit le contexte. L'indétermination est ici à distinguer de la polysémie, au sens de multiplicité des acceptions pour une même entrée terminologique :

Vague concepts have a large extensional domain of indeterminacy. An extensional domain of indeterminacy is a domain where it is sometimes impossible to decide whether a given referent is a member of the extension of the concept or not, even in context. These concepts always have low determination. (Andersen $2002: 139$ )

\subsection{Une illustration : le terme chemical species}

En guise d'illustration, le terme chemical species, concept fondamental et historique en chimie organique, fait l'objet des définitions suivantes :

(1) An ensemble of chemically identical molecular entities that can explore the same set of molecular energy levels on the time scale of the experiment. The term is applied equally to a set of chemically identical atomic or molecular structural units in a solid array. [...] Except where the context requires otherwise, the term is taken to refer to $a$ set of molecular entities containing isotopes in their natural abundance. The wording of the definition given in the first paragraph is intended to embrace both cases such as graphite, sodium chloride or a surface oxide, where the basic structural units may not be capable of isolated existence, as well as those cases where they are. In common chemical usage, generic and specific chemical names (such as radical or hydroxide ion) or chemical formulae refer either to a chemical species or to a molecular entity. (IUPAC, 1994: 66, 1077 (Glossary of terms used in physical organic chemistry [IUPAC Recommendations 1994] : 1096)

(2) Although this noun has a precise meaning in some disciplines, such as in biology, in chemistry-speak it is used as an imprecise, but convenient term, to denote either atoms, ions, radicals, intermediates, fragments of molecules, or other chemical entities sometimes of uncertain or imprecisely defined structure. (The Vocabulary and Concepts of Organic Chemistry 2005)

(3) Atoms, molecules, molecular fragments, ions, etc., being subjected to a chemical process or to a measurement. Generally, a chemical species can be defined as an ensemble of chemically identical molecular entities that can explore the 
same set of molecular energy levels on a characteristic or delineated time scale. The term may be applied equally to a set of chemically identical atomic or molecular structural units in a solid array. (Wikipedia 2012)

D'aucuns constateront d'emblée un manque de consensus définitoire évident entre des énoncés qui, s'ils sont issus de sources dictionnairiques variées, relèvent néanmoins du même domaine et sous-domaine de spécialité (en l'occurrence les mécanismes réactionnels), ce qui exclut par conséquent les nuances sémantiques intrinsèques à la notion de domaine. Aucune de ces définitions ne procure une description précise et arrêtée du terme traité, ce qui se révèle problématique tant au niveau de la structuration notionnelle du domaine que de la transmission des connaissances. La variabilité des définitions présentées ci-dessus constitue donc incontestablement un premier signe d'indétermination terminologique.

On observe également, dans un deuxième temps, le recours massif à ce que Vladimir Leitchik et Serguei Shelov (2007: 99) appellent des indices polymorphiques². Il s'agit d'un ensemble de formulations, d'adverbes ou de conjonctions qui limitent la portée assertive et donc universelle de la définition, à l'instar des locutions suivantes : is taken to refer to ; is applied equally to ; the wording of the definition is intended to embrace both [...], in common chemical usage; or ; either, etc.

définition issue du Vocabulary and Concepts of Organic Chemistry (VCOC) constitue un véritable constat d'échec définitoire et terminologique dans la mesure où il ne s'agit pas d'une véritable définition, mais d'une sorte de note explicative dans laquelle les auteurs justifient l'absence de définition par l'imprécision conceptuelle caractérisant le terme décrit. L'énumération qu'ils font des entités chimiques pour tenter de qualifier le terme s'apparente partiellement à une définition par extension. Mais, en réalité, ces entités chimiques ne sont en aucun cas liées les unes aux autres par un lien de généricité.

Comme annoncé, l'analyse définitoire s'accompagne d'une décomposition sémantique de chacune des unités en hyperonyme, traits constitutifs et propriétés (cf. Tableau 2).

Tableau 2. Décomposition sémantique comparée du terme chemical species

\begin{tabular}{|c|c|c|c|}
\hline & IUPAC & $\mathrm{VCOC}$ & Wikipedia \\
\hline Hyperonyme & Ensemble & $?$ & $?$ \\
\hline $\begin{array}{l}\text { Traits } \\
\text { constitutifs }\end{array}$ & $\begin{array}{l}\text { - molecular } \\
\text { entities } \\
\text { - isotopes }\end{array}$ & $\begin{array}{l}\text { - atoms } \\
\text { - ions } \\
\text { - fragments of } \\
\text { molecules } \\
\text { - radicals } \\
\text { - intermediates } \\
\text { - other chemical } \\
\text { entities }\end{array}$ & $\begin{array}{l}\text { - atoms } \\
\text { - ions } \\
\text { - molecules } \\
\text { - molecular fragments }\end{array}$ \\
\hline Propriétés & - solid array & - imprecise structure & $\begin{array}{l}\text { - subjected to a chemical process or to a } \\
\text { measurement }\end{array}$ \\
\hline
\end{tabular}



(radicaux, molécules, complexes, groupes fonctionnels, acides, etc.) différentes les unes des autres, ce qui représente un obstacle majeur à la constitution de classes d'objets plus fines issues du regroupement de caractéristiques communes :

Moreover, some compounds with more than 4 carbon atoms are also treated. The oxidation pathways of 34 chemical species ( 5 alcohols, 10 carbonyl compounds, 13 mono-and dicarboxylic acids, 1 ester, 4 polyfunctional compounds and 1 heterocyclic compound) were included and critically reviewed (Table I, ESM). (CS) An activated complex is the chemical species present at the transition state of a reaction. (CP)

A radical is a chemical species that has a single unpaired electron in one of its orbitals. (CP)

George Hammond formulated a useful principle that relates the nature of a transition state to its location on the reaction path. This Hammond Postulate states that a transition state will be structurally and energetically similar to the species (reactant, intermediate or product) nearest to it on the reaction path. (CP)

A novel species, carbon-centered tri-s-tetrazine $(\mathrm{C} 4 \mathrm{~N} 9 \mathrm{H} 3)$, and its 10 derivatives (C4N9-R3, where $\mathrm{R}=\mathrm{OH}, \mathrm{F}, \mathrm{CN}, \mathrm{N} 3, \mathrm{NH} 2, \mathrm{NO} 2, \mathrm{~N} \mathrm{NH}, \mathrm{N} 2 \mathrm{H} 3, \mathrm{CBB} \mathrm{CH}$, and $\mathrm{CH} \mathrm{CH} 2$ ) have been studied computationally (CS)

At present, the largest organic species clearly identified in interstellar icy grains is $\mathrm{CH} 3 \mathrm{OH}$ [23]. Laboratory work is currently underway to identify the many weak absorption bands that are possibly due to organic species (e.g. formic acid, formamide and acetaldehyde). (CS)

\subsection{Synthèse}

À l'instar de chemical species, il a été clairement établi qu'une grande partie des termes $\mathrm{du}$ domaine sont non seulement imprécis sur un plan définitoire ou conceptuel, mais qu'ils ne se prêtent guère à une approche conceptuelle et logique des unités linguistiques, autrement dit à une caractérisation des concepts par le biais de classes d'objet et de traits distinctifs. Ce constat s'applique aussi bien aux termes génériques de la chimie organique (atom, chemical element, functional group, molecule) qu'aux unités dénotant des réactions organiques spécifiques (addition, oxydation, reduction, substitution). Néanmoins, nous avons également observé que cette indétermination semble, à certaines reprises, constituer un pis-aller, voire une commodité terminologique, pour les chimistes organiciens. En effet, la complexité du domaine est telle qu'une application rigide des normes terminologiques est inenvisageable et non opératoire en l'état. La problématique de transmission des connaissances spécialisées n'en demeure pas moins opportune.

\section{Phénomènes linguistiques de compensation}

En réalité, une analyse approfondie du corpus, et plus particulièrement des variantes terminologiques (Daille et al. 2000), d'une part, et des syntagmes de longueur deux à 
cinq (Frantzi, et al. 1999 ; Ormrod 2001), d'autre part, a permis de constater que cette indétermination est partiellement et principalement compensée par trois phénomènes.

\subsection{La représentation figurative}

28 Le premier de ces phénomènes, qui caractérise également d'autres disciplines technoscientifiques, est le recours aux équations chimiques et aux représentations figuratives de toutes sortes (graphiques, symboles mathématiques, schémas moléculaires, formules stéréochimiques, données chiffrées, etc.). L'utilisation de cet autre système sémiotique permet non seulement de répondre à une volonté d'économie de langage, caractéristique des publications scientifiques, mais il satisfait également un besoin de clarification face à des données particulièrement complexes :

La représentation graphique d'un objet est souvent irremplaçable. Pour faire comprendre ce qu'est un outil [...], un dessin fera gagner du temps. À plus forte raison, là où une description en langue naturelle aura bien du mal à rendre compte de ce qu'est un vérin, un dessin industriel correct, assisté ou non, fera voir de quoi il s'agit. (Lerat $1995: 149)$

Les équations chimiques et les schémas moléculaires sont reconnus sur un plan international, de même que l'étaient les symboles alchimiques qui les ont précédés. Ces supports ont donc leur propre champ d'application et dépassent largement la communication linguistique. Néanmoins, ainsi que le signale F. Gaudin (2003: 66), ce code figuratif trouve ses limites et génère des ambiguïtés dès lors qu'il s'agit de déduire des informations plus précises sur les substances, et plus particulièrement sur les molécules ainsi représentées (nombre d'électrons, etc.). En effet, le souci de concision et d'explicitation (associée à l'extrême complexité des données chimiques) donne lieu à une forme d'approximation au sein des équations et des représentations moléculaires. À titre d'exemple, les représentations spatiales de molécules en autant d'atomes et de liaisons chimiques (représentation héritée du principe de Pauli en physique quantique), c'est-à-dire les représentations à trois dimensions, correspondent à des schématisations plus ou moins approximatives de la réalité :

De la même façon que les frontières de la France peuvent se schématiser sous forme d'un hexagone, l'espace occupé par les électrons autour d'un noyau d'atome ressemble peu ou prou à une sphère. Dans les deux cas, cette forme approximative représente l'objet lui-même. Les représentations conventionnelles à trois dimensions de molécules chimiques sont donc fondées sur une métonymie de la propriété pour l'objet. (Fries 2005 : 234)

De même, dans les équations chimiques développées, les atomes sont désignés par des acronymes (correspondant aux éléments chimiques) qui représentent en réalité un nombre macroscopique d'atomes par lettre et non un seul atome, comme semble le faire croire l'équation (qui s'apparente dans ce cas à une synecdoque). Enfin, paradoxalement, malgré ces approximations, l'ensemble de ces illustrations est uniquement compréhensible des initiés, voire des spécialistes pour les plus complexes d'entre elles. Cette opacité témoigne une nouvelle fois de l'implicite entre experts d'une même discipline, ce qui constitue une difficulté d'appréhension du domaine supplémentaire. 


\subsection{Liberté combinatoire}

En second lieu, l'indétermination terminologique est compensée par une liberté combinatoire, syntaxique et néologique extrêmement forte de la part des spécialistes organiciens. Les chimistes exploitent pleinement les modes de composition par juxtaposition, par apposition ou par concaténation et les modes de dérivation propres à la langue anglaise. Ils contredisent ainsi le "tout" substantif et dénominatif qui caractérise a priori les terminologies des disciplines technoscientifiques. La pluralité des constructions syntaxiques relevées dans le corpus témoigne de cette liberté combinatoire (voir tableaux 3a et 3b).

Tableau 3a. Constructions syntaxiques relevées dans le corpus

\begin{tabular}{|l|l|}
\hline acid-catalyzed ring opening reaction & chain-breaking reaction \\
alkyl bromide substitution reaction & disrotatory electrocyclic reaction \\
aldol-like condensation reaction & enzyme-catalyzed reaction \\
aliphatic nucleophilic substitution & gallium-mediated reaction \\
reaction & intramolecular ring-opening reaction \\
alternative radical chain-reaction & nucleophile-electrophile reaction \\
aqueous enzymatic reaction & enantioselective photochemical reaction \\
aromatic electrophilic substitution & nonsolvolytic bimolecular reaction \\
reaction & nucleophile-electrophile bonding reaction \\
asymmetric organocatalysed reaction & reversible intramolecular epoxide opening reaction, \\
catalytic enantioselective reaction & etc. \\
\hline
\end{tabular}

Tableau 3b. Variantes syntaxiques d'insertion

\begin{tabular}{|l|l|}
\hline \multicolumn{1}{|c|}{ Terme de base } & \multicolumn{1}{c|}{ Variantes } \\
\hline aldol reaction & $\begin{array}{l}\text { aldol-like reaction } \\
\text { aldol type reaction } \\
\text { aldol-type reaction }\end{array}$ \\
\hline Mannich reaction & $\begin{array}{l}\text { Mannich-like reaction } \\
\text { Mannich-type reaction }\end{array}$ \\
\hline Reformatsky reaction & $\begin{array}{l}\text { Reformatsky like reaction } \\
\text { Reformatsky-like reaction } \\
\text { Reformatsky-type reaction } \\
\text { (reaction in a Reformatsky-like fashion) }\end{array}$ \\
\hline
\end{tabular}

Dans le tableau $3 \mathrm{~b}$, l'insertion d'un simple mot grammatical permet au chimiste d'introduire une forme de distanciation par rapport à la notion de départ et induit, en l'occurrence, la création d'un nouveau concept. En effet, l'inclusion au sein du syntagme des éléments grammaticaux like et type permet d'indiquer aux locuteurs que 
les réactions ainsi décrites se distinguent plus ou moins fortement du mécanisme initial, soit en raison d'un mode opératoire divergent ou d'étapes réactionnelles inversées, soit en raison de l'utilisation de substances chimiques inhabituelles. Il est à noter que lorsque la nuance n'est pas précisée par le spécialiste, le terme s'accompagne systématiquement d'équations ou de schémas numérotés, qui compensent le sémantisme parfois large de ces nouvelles expressions, ainsi qu'illustré dans le corpus :

DMF can also work as electrophile with the zinc-based Reformatsky agent derived from ethyl chlorodifluoroacetate, thus giving a Vilsmeier-type $\mathrm{N}-\mathrm{O}$ acetal product 304 , convertible into the respective ethyl hemiacetal 305, which is chemically equivalent to a difluoromalonaldehydic derivative 306. This latter product condenses with active methylene compounds via aldol-like reactions (Eq. 87), 319 and the procedure is useful for the synthesis of diverse functionalized a, adifluorinated esters and amides. (CS)

The other metal-catalyzed reactions with five-membered pincers are asymmetric aldol-type condensations [215, 224, 226, 227], cyclo-propanations [229], enantioselective allylations [230], reductive eliminations [231], transfer hydrogenations [214, 215, 227, 228], hydroaminations [215], polymerizations [215], etc. [224, 232]. (CS)

The first Mukaiyama aldol-type reaction catalysed by lanthanides derivatives was described by Kagan et al. 31 with the presence of 5 mol\% of several lanthanide trichlorides. The reactions were rather slow ( $12 \mathrm{~h}$ to several days) and yielded either O-silylated or desilylated aldols. A few years later, Collin et al. showed the efficiency of lanthanide iodides as catalysts in Mukaiyama aldol-type reaction. 32 The reactions are very fast $\left(5 \mathrm{~min}\right.$ at $\left.2788^{\circ} \mathrm{C}\right)$ and yields are excellent. (CS)

Enfin, nous attirons également l'attention sur la construction « in a Reformatsky-like fashion» qui correspond au degré le plus élevé de variation et de reformulation employé par les spécialistes et dont on retrouve un certain nombre de déclinaisons dans le corpus :

reaction in a switch-like fashion; reaction (continues) in a chain-like fashion; reaction (proceeds) in a zipper-like fashion; reaction (progresses) in a step-like fashion ; reaction (propagates) in a wave-like fashion ; reaction (would proceed) in a glutamate-dehydrogenase-like fashion, etc.

En conclusion, l'étude des variantes terminologiques a permis de révéler que les chimistes organiciens n'hésitaient pas à introduire au sein de concepts déjà existants des modifications formelles et sémantiques plus ou moins importantes, génératrices ou non de nouvelles unités, mais qui témoignent dans tous les cas d'une prise de liberté rédactionnelle et terminologique significative de leur part.

\subsection{Créativité terminologique par incrémentation}

Enfin, l'indétermination terminologique est également et avant tout compensée par une structuration conceptuelle extrêmement dense et par une construction sémantique et syntaxique des termes par «stratification » :

- alkylation (reaction)

- allylic alkylation

- asymmetric allylic alkylation

- catalytic asymmetric allylic alkylation

- intramolecular asymmetric allylic alkylation

- sequential asymmetric allylic alkylation

- copper-catalyzed asymmetric allylic alkylation 
- copper-free asymmetric allylic alkylation

- cuprate-mediated asymmetric allylic alkylation

- molybdenum-catalyzed asymmetric allylic alkylation

- palladium-catalysed asymmetric allylic alkylation

- Pd-catalysed asymmetric allylic alkylation

- palladium-catalyzed decarboxylative asymmetric allylic alkylation

- transition-metal-catalyzed asymmetric allylic alkylation, etc.

Dans la série entourant le terme alkylation, les experts font appel à un mode de créativité terminologique par incrémentation (Portelance 1987: 359). À chaque nouvelle unité de la série, correspond un nouvel ajout d'information, chaque détermination supplémentaire étant différentielle. L'utilisation d'éléments sémantiques forts à l'image des adjectifs permet de compenser le caractère extrêmement vague des termes génériques qui sont à la base du syntagme. Ce système est, à notre sens, extrêmement révélateur de la dynamique du vocabulaire technoscientifique en langue anglaise, une dynamique qui repose à la fois sur le potentiel combinatoire des unités composant les nomenclatures et sur leur potentiel taxinomique. Il permet en effet de générer des séries de syntagmes à noyau commun parfois extrêmement développées et potentiellement de longueur infinie puisque chacune de ces nominalisations permette d'organiser les objets dans plusieurs sousclassifications.

Ces constructions par stratification indiquent également que les scientifiques communiquent souvent leur savoir commun dans un langage qui montre la complexité croissante de leurs connaissances. Pourtant, selon certains linguistes (Ormrod 2001 : 10 ; Soubrier 2005 : 291), ce mode de dénomination par incrémentation peut également engendrer des problèmes de lisibilité et de compréhension conceptuelle. En effet, plus le composé est long et/ou technique, plus il devient difficile à déchiffrer pour les néophytes, contrairement au français qui, lui, est contraint d'utiliser une construction prépositionnelle pour décrire le concept. Ainsi, d'après Jean Soubrier, les constructions syntaxiques françaises, quoique moins économiques, permettent en revanche de clarifier certaines ambiguïtés des termes anglais tout en améliorant la lisibilité. La concision anglaise s'obtiendrait donc, selon le linguiste, au détriment de la transparence conceptuelle. Cette opinion n'est toutefois pas partagée par christine Portelance (1987 : 359) qui affirme, au contraire, que la longueur et la technicité de ces syntagmes seraient compensées par la «capacité de la mémoire de compréhension » qui agit dans toute nomenclature. Le terme de base, à condition d'être suffisamment explicite, permet de créer des combinaisons également explicites par le biais d'unités lexicales elles-mêmes identifiables grâce à la mise en contexte des termes et grâce à l'ensemble des liens logiques et indirects existants entre ces unités :

Avec une telle capacité générative, toute notion élaborée dans une discipline pourra être nommée et distinguée des autres notions par le jeu des références et des oppositions selon le même principe qui permet à un système linguistique d'engendrer un nombre infini de phrases. (Portelance $1987: 359$ )

\section{Multidimensionnalité}

Seulement, ce fonctionnement par stratification est également à l'origine de la multidimensionnalité latente du domaine et donc de nombreuses difficultés de classification notionnelle. Par multidimensionnalité, nous entendons un phénomène de 
classification conceptuelle qui apparaît lorsque des concepts sont classés de plusieurs manières au sein d'un système notionnel. Les caractéristiques distinctives et les relations conceptuelles sont les deux principaux facteurs à l'origine de la multiplicité d'approches d'un même concept (Bowker \& Meyer 1993 ; Bowker 1997), ainsi qu'illustré dans les syntagmes suivants :

- asymmetric organocatalysed reaction

- alternative radical chain-reaction

- chain-growth polymerization reaction

- electrophilic aromatic substitution reaction

- biochemical carbonyl condensation reaction

- carbon-carbon bond-forming reaction

- asymmetric aldol reaction

- ammonia-chlorine reaction

- alkyl halide substitution reaction

- carboxylic acid reaction, etc.

L'observation des classes sémantiques auxquelles appartiennent les éléments lexicaux figurant dans les nominalisations relevées ci-dessus atteste à la fois de la multiplicité et de la superposition de plusieurs dimensions au sein d'un même syntagme puisqu'on décèle simultanément des éléments relevant des mécanismes réactionnels au sens large (condensation, polymerization, substitution, etc.), ceux décrivant les types de processus réactionnels mis en œuvre (aromatic, asymetric, biochemical, chain-growth, coupling, electrophilic, organocatalysed, radical, etc.) et enfin des éléments renvoyant à des composés organiques (alkane, alkene, aldol, ammonia-chlorine, carbon, carbonyl, carboxylic acid, etc.).

À l'image de la multidimensionnalité décrite par Kyo Kageura (1997:122), un même objet peut être désigné dans un texte selon plusieurs perspectives, si diverses caractéristiques du concept représentant cet objet sont activées ou si différentes relations envers d'autres concepts sont établies. Or, cette incidence de la caractéristique/relation peut avoir lieu à tous les niveaux du réseau puisqu'en fonction de la perspective adoptée, c'est-à-dire de la dimension ou de la facette activée au sein du syntagme, la nature des liens sémantiques et donc la physionomie du réseau notionnel s'en trouveront modifiées. Elle induit donc des phénomènes de polyhiérarchie. Dans le cas présent, les différentes facettes des concepts chimiques sont activées simultanément et s'interpénètrent, ce qui rend toute tentative de représentation graphique périlleuse, tout en imposant au terminologue de privilégier de façon relativement arbitraire une dimension à l'exclusion des autres.

41 Enfin, les phénomènes de multidimensionnalité au sein de la chimie sont également liés à la multiplicité des mécanismes réactionnels pouvant mener à une même réaction. En effet, une même réaction ou un même produit chimique peut être obtenu non seulement par le biais d'un ordre d'étapes mécanistiques successives divergeant, mais également en combinant des réactions et des espèces chimiques différentes. Ainsi, dans l'arborescence donnée en figure 1, nous voyons clairement que les deux concepts halogenation et Wurth reaction peuvent être placés indifféremment à deux endroits différents du réseau notionnel, ce qui explique le caractère extrêmement vague de certaines définitions des principales réactions organiques. 


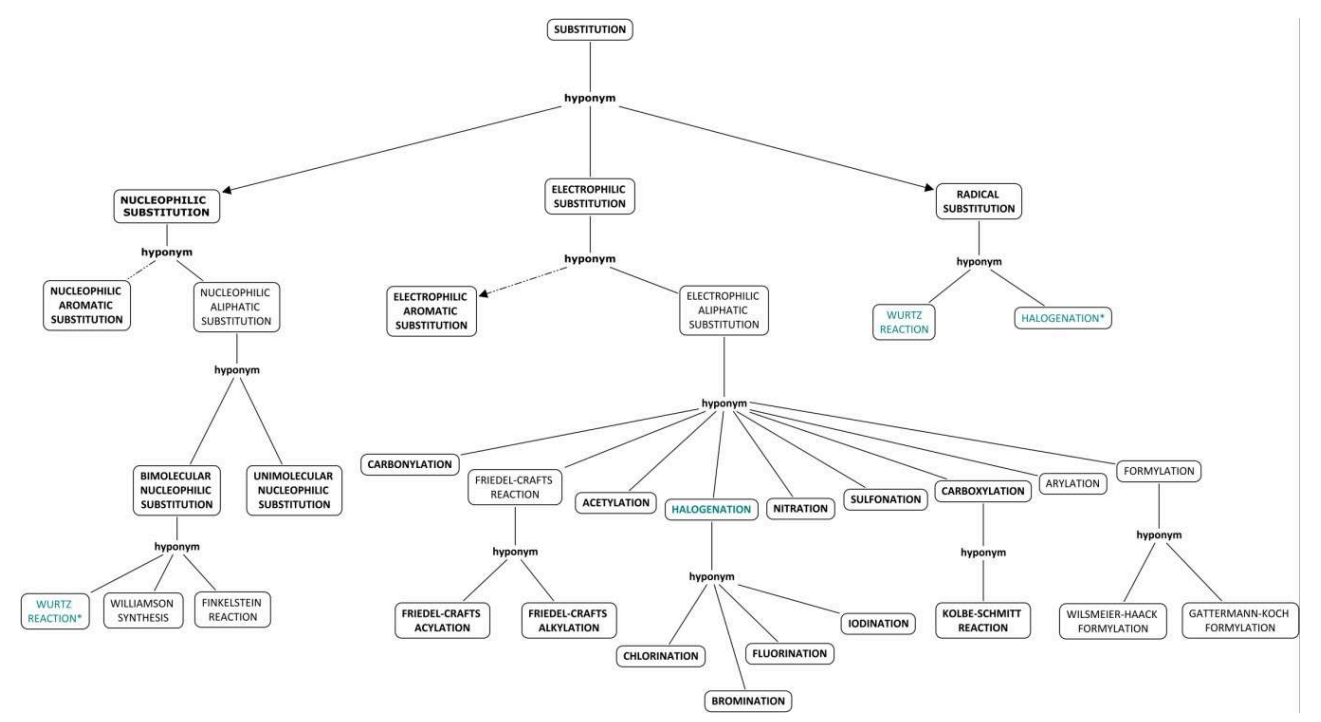

\section{Conclusion}

L'étude de l'indétermination terminologique et de la multidimensionnalité nous a permis de mettre à jour un certain nombre de spécificités à la fois d'ordre lexical et morphosyntaxique propres à l'anglais de la chimie.

Parmi ces spécificités, nous avons mis en évidence le potentiel de nomination et de structuration des lexiques technico-scientifiques. Nous avons constaté en effet que la structure principale des syntagmes relevés associait systématiquement un ou plusieurs déterminants et un noyau indiquant la classe conceptuelle à laquelle appartient le terme. Ces combinaisons autorisent par conséquent un système de structuration hiérarchique parfois extrêmement dense. Un tel mode de construction syntagmatique est somme toute relativement classique dans la mesure où il correspond à l'approche empiro-inductive qui caractérise habituellement le raisonnement anglo-saxon. Néanmoins, il permet de contredire largement le fait que la majorité des termes complexes soient des composés binominaux ou binaires, ainsi que le soulignent Béatrice Daille, Benoît Habert, Christian Jacquemin et Jean Royauté (2000) ou encore Katarina Frantzi, Sophia Ananiadou et Jun-ichi Tsujii (1999), qui vont jusqu'à affirmer que ces derniers constituent près de $90 \%$ des unités composées. chimie sont indéterminés et multidimensionnels, ce qui constitue un frein en matière d'appréhension et de transmission des connaissances pour les apprenants de cette discipline. C'est pourquoi l'un des versants appliqués de ce projet de recherche fut de proposer une ébauche de ressource onto-terminologique, fondée notamment sur l'exhaustivité des champs terminologiques et sur une adaptation au domaine de la chimie afin de pallier les faiblesses de l'approche terminologique classique et de la définition intensionnelle.

Toutefois, au-delà de ces difficultés, nous nous apercevons également que l'indétermination et la multidimensionnalité font partie intégrante des éléments de construction du savoir en chimie. Il semblerait, en effet, que paradoxalement les chimistes organiciens cultivent une forme d'imprécision autour de certains concepts 
notamment génériques, afin de gérer l'extrême complexité des processus réactionnels à décrire. En d'autres termes, ils font de ces approximations conceptuelles une commodité langagière.

\section{BIBLIOGRAPHIE}

Andersen, Øivin. 2002. «Terminology and pragmatics ». In Koskeia M., C. Lauren, M. Nordman et N. Pilke (dir.), Porta Scientiae I. Lingua specialis. Vaasa : Proceedings of the University of Vaasa, 136-147.

Andersen, Øivin. 2007. «Indeterminacy, context, economy and well-formedness in specialist communication ». In Antia, B. (dir.), Indeterminacy in LSP and Terminology. Amsterdam/ Philadelphie : John Benjamins, 3-14.

Assal, Allal. 1994. «La notion de notion en terminologie ». Meta : journal des traducteurs/Meta: Translators' Journal 39/3, 460-464.

Aussenac-Gilles, Nathalie et Anne Condamines. 2009. « Variation syntaxique et contextuelle dans la mise au point de patrons de relations sémantiques ». In Minel J.-L. (dir.), Filtrage sémantique. Paris : Hermes/Lavoisier, 115-149.

Béjoint, Henri. 1993. «La définition en terminographie ». In Arnaud P. et P. Thoiron (dir.), Aspects du vocabulaire. Lyon : Presses Universitaires de Lyon, 19-26.

Bowker, Lynne et Ingrid Meyer. 1993. «Beyond 'textbook' concept systems: handling multidimensionality in a new generation of term banks ». In TKE'93: Terminology and Knowledge Engineering. Proceedings of the Third International Congress on Terminology and Knowledge Engineering. Francfort : Indeks Verlag, 123-137.

Bowker, Lynne. 1997. « Multidimensional classification of concepts and terms ». In Wright S. E. et G. Budin (dir.), Handbook of Terminology Management. Volume I. Amsterdam/Philadelphie : John Benjamins, 133-143.

Bühler, Karl. 1983 [1934]. Sprachtheorie. Die Darstellungsfunktion der Sprache. Stuttgart : Gustav Fischer.

Candel, Danielle. 2000. « La définition chez les scientifiques ». Terminologies Nouvelles 21, 52-57.

Candel, Danielle et alii. 2001. « Aspects du groupe nominal dans l'activité définitoire des scientifiques ». Revue française de linguistique appliquée 6, 17-28.

Condamines, Anne et Josette Rebeyrolle. 2000. «Construction d'une base de connaissances terminologiques à partir de textes : expérimentation et définition d'une méthode ». In Charlet J., M. Zacklad, G. Kassel et D. Bourigault (dir.), Ingénierie des connaissances, évolutions récentes et nouveaux défis. Paris : Eyrolles, 225-242.

Daille, Béatrice, Benoît Habert, Christian Jacquemin et Jean Royauté. 2000. « Empirical observation of term variations and principles for their description ». Terminology 3/2, 197-257.

Dehne, G. Clarck. 1997. « Alan D. McNaught and Andrew Wilkinson, compilers. Compendium of chemical terminology, IUPAC recommendations ». Terminology 4/2, 347-351. 
Dréan, Gérard. 2001. «Comment enseigner l'économie? ». Sociétal 32, accessible sur $<$ www.perso.club-internet.fr/gdrean>.

Enguehard, Chantal. 2005. « Un banc de test pour la reconnaissance de termes en corpus ». In Williams G. (dir.), La linguistique de corpus. Rennes : Presses Universitaires de Rennes, 273-286.

Frantzi, Katarina T., Sophia Ananiadou \& Jun-ichi Tsujii. 1999. « Automatic classification of technical terms using the NC-value method for term recognition ». In Proceedings of the 5th Conference on Computational Lexicography and Text Research, Pecs, Hongrie, juin 16-19 1999, 57-66.

Freixa, Aymerich, Judit Sabela Fernandez Silva et Maria Teresa Cabre Castellvi. 2008. « La multiplicité des chemins dénominatifs ». Meta : journal des traducteurs/Meta: Translators' Journal 53/4, 731-747.

Fries, Marie-Hélène. 2005. «Les figures de rhétorique dans les articles de chimie : un révélateur du contexte en recherche scientifique ». Bulletin de la société de stylistique anglaise 26, 227-249.

Gambier, Yves. 1991. «Présupposés de la terminologie : vers une remise en cause ». Cahiers de linguistique sociale 18, 31-58.

Gaudin, François. 2003. Socioterminologie : une approche sociolinguistique de la terminologie, $1^{\text {re }}$ éd., coll. Manuels champs linguistiques. Louvain-la-Neuve : Duculot/De Boeck.

Geeraerts, Dirk et alii. 1994. The Structure of Lexical Variation. Meaning, Naming and Context. Berlin/New York : Mouton de Gruyter.

Godly, Ted. 1993. "Terminological principles and methods in the subject field of chemistry". In Sonneveld H. B. et K. L. Loening (dir.), Terminology: Applications in interdisciplinary communication. Amsterdam/Philadelphie : John Benjamins, 141-164.

Jacques, Marie-Paule. 2005. «Pourquoi une linguistique de corpus?». In Williams G. (dir.), La linguistique de corpus. Rennes : Presses Universitaires de Rennes, 21-30.

Kageura, Kyo. 1997. « Multifaceted/multidimensional concept systems ». In Wright, S. E. \& G. Budin (dir.), Handbook of Terminology Management. Volume I. Amsterdam/Philadelphie : John Benjamins, 119-132.

Krief, Alain et Catherine Castillo-Collaux. 2005. « EnCOrE (Encyclopédie de chimie organique électronique): An original way to represent and transfer knowledge from freshmen to researchers in organic chemistry ». In Electronic Workshops in Computing. 3rd International LeGEWG Workshop: GRID Infrastructure to Support Future Technology Enhanced Learning, Berlin, 3 décembre 2003.

Krief, Alain, Catherine Castillo-Collaux, Julie Henry, Claudie Juilliard et Sandrine Peraldi. 2008. "Building the EnCOrE Dictionary collaboratively: Strategy and practice". International Journal of Knowledge and Learning 4/2-3, 203-216.

Lefèvre, Michel. 2004. « Terminologie et discours 'empratique' ». In Cortès C. (dir.), Cahiers du C.I.E.L : Des fondements théoriques de la terminologie, Université Paris 7, 53-70.

Leitchik, Vladimir M. and Serguey D. Shelov. 2007. « Commensurability of scientific theories and indeterminacy of terminological concepts ». In Antia B. (dir.), Indeterminacy in LSP and Terminology. Amsterdam/Philadelphie : John Benjamins, 93-106.

Lerat, Pierre. 1995. Les langues spécialisées. Paris : Presses Universitaires de France.

L'homme, Marie-Claude. 2004. Terminologie : principes et techniques. Montréal : Presses de l'Université de Montréal. 
Loening, Kurt L. 1986. «Terminological guidelines and their application in the International Union of Pure and Applied Chemistry (IUPAC) ». In Krommer-Benz M. (dir.), International Cooperation in Terminology Work. INFOTERM Series 8. K. G. Saur : Munich, 92-116.

Ormrod, Janet. 2001. «Construction discursive de noms composés dans des textes scientifiques anglais ». In Banks D. (dir.), Le groupe nominal dans le texte spécialisé. Paris : l'Harmattan, 9-23.

Otman, Gabriel. 1996. Les représentations sémantiques en terminologie. Paris : Masson.

Portelance, Christine. 1987. « Fertilisation terminologique ou insémination terminologique artificielle ». Meta : journal des traducteurs/Meta: Translators' Journal 32/3, 356-360.

Pearson, Jennifer. 1998. Terms in Context. Studies in Corpus Linguistics 1. Amsterdam : John Benjamins.

Rey-Debove, Josette. 1971. Étude linguistique et sémiotique des dictionnaires français contemporains. La Hague/Paris : Mouton.

Rogel, Thierry. 2007. « Durcir les 'sciences molles', mollir les 'sciences dures' ». In SES, IUFM d'Aix-Marseille. Accessible sur <http://www.aix-mrs.iufm.fr/formations/filieres/ses/didac tique/rogel.pdf>.

Sager, Juan C. 1990. A Practical Course in Terminology Processing. Amsterdam/Philadelphie : John Benjamins.

Soubrier, Jean. 2005. « Transparence et opacité de l'anglais scientifique ». In Béjoint, H. et Maniez F. (dir.), De la mesure dans les termes, Travaux du CRTT. Lyon : Presses Universitaires de Lyon, 280-295

Swales, John M. 1990. Genre Analysis. English in academic and research settings. Cambridge : Cambridge University Press.

Temmerman, Rita. 2000. Towards New Ways of Terminology Description. The Sociocognitive Approach. Amsterdam/Philadelphie : John Benjamins.

Thoiron, Philippe, Pierre Arnaud, Henri Béjoint et Claude-Pierre Boisson. 1996. « Notion d"archiconcept' et dénomination ». Meta : journal des traducteurs/Meta: Translators' Journal 41/4, 512- 524.

Weissenhofer, Peter. 1995. Conceptology in Terminology Theory, Semantics and Word-formation: A morpho-conceptually based approach to classification as exemplified by the English baseball terminology. Vienne : TermNet.

Wüster, Eugen. 1968. Dictionnaire multilingue de la machine-outil. Volume de base Anglais Français. Londres : Technical Press.

\section{NOTES}

1. En effet, l'identité d'un concept n'équivaut jamais à la somme de ses traits conceptuels dans la mesure où il est impossible de représenter parfaitement la réalité. En l'occurrence, comme le signale Gabriel Otman (1996: 12), la décomposition des unités terminologiques en unités minimales de sens pose également le problème du « choix des traits sémantiquement pertinents et des unités indécomposables de la langue. "

2. Les indices figurent en italique dans les énoncés définitoires. 


\section{RÉSUMÉS}

Cet article porte sur une langue de spécialité relativement peu étudiée en anglais : celle de la chimie organique. Le discours de spécialité chimique est analysé à travers la mise en évidence de deux phénomènes linguistiques également méconnus : l'indétermination terminologique et la multidimensionnalité dans les sciences exactes. En effet, malgré la présence d'une nomenclature en chimie, une exploration textuelle outillée d'un double corpus, associée à une analyse componentielle revisitée des énoncés définitoires de certains termes clés a permis d'établir clairement que ces derniers étaient non seulement imprécis, mais qu'ils ne se prêtaient pas à une approche conceptuelle et logique des unités linguistiques. L'auteur montre également que cette indétermination est partiellement compensée par une très forte créativité lexicale, une structuration conceptuelle extrêmement dense et une construction sémantique des termes par incrémentation. Néanmoins, ce fonctionnement par stratification est également à l'origine de la multidimensionnalité latente du domaine et donc des nombreuses difficultés de classification et de représentation notionnelles de la discipline.

This paper focuses on a relatively unexplored field of research within English for Specific Purposes: that of organic chemistry. The specialized discourse of chemistry is analyzed through the identification of two specific linguistic phenomena that are also often overlooked: terminological indeterminacy and multidimensionality within exact sciences. Indeed, despite the existence of a chemical nomenclature, the exploration (through a concordancer) of a double corpus combined with a fresh perspective on the componential analysis of certain definitions and a semantic breakdown around key terms shows that certain terminological units are imprecise and do not allow for a conceptual and ontological approach. The author also shows that this terminological and notional indeterminacy is partly offset by a very strong lexical creativity, an extremely dense conceptual organization and layered semantic constructions. However, these phenomena also lead to strong multidimensionality within the field and many difficulties regarding conceptual organization and representation.

\section{INDEX}

Mots-clés : analyse définitoire, base de connaissances, chimie organique, indétermination, multidimensionnalité, terminologie textuelle, variation terminologique

Keywords : defining analysis, indeterminacy, knowledge base, multidimensionality, organic chemistry, terminological variation, textual terminology

\section{AUTEUR}

\section{SANDRINE PERALDI}

Sandrine Peraldi est responsable de la recherche au sein de l'ISIT pour la filière Communication interculturelle et traduction. Membre du CRATIL (ISIT) et du CLILLAC-ARP (Paris Diderot), sa thèse de doctorat, soutenue à l'Université Paris Diderot, est intitulée « Indétermination terminologique et multidimensionnalité dans le domaine de la chimie organique ». Elle s'intéresse à l'analyse des discours scientifiques et techniques en langues anglaise et française et au fonctionnement des unités spécialisées en contexte. Elle étudie en particulier la structuration conceptuelle des disciplines technoscientifiques ainsi que les phénomènes de variation, de 
néologie, de multidimensionnalité et d'indétermination terminologiques, par le biais d'une exploration et d'une analyse outillée sur corpus. s.peraldi@isit-paris.fr 